\title{
A note on the convergence of linear semi-groups of class $(1, \mathrm{~A})$
}

\author{
Nazar H. ABDELAZIZ* \\ (Received June 22, 1988, Revised December 26, 1988)
}

\begin{abstract}
In the present paper we prove a Trotter-Kato type convergence theorems for semi-groups of linear operators of class $(1, A)$. Our results genaralize those of I. Miyadera [5]
\end{abstract}

\section{$\S 0$. Introduction}

The present paper deals with the problem of convergence of semigroups of linear operators, that is, determining wether or not a given sequence of semi-groups converge in some sense to a semi-group. This problem was investigated by many authors in the case of a sequence of $C_{0}$ semi-groups $T_{n}(t)$ satisfying the condition

$$
\left\|T_{n}(t)\right\| \leq M e^{\omega t}, n \in N,
$$

where $M$ and $\omega$ are independent of $n$ (cf. [1], [3], [4], [6], [8]). Miyadera [5] also treated the problem under somewhat more general conditions and for a more general class $(1, A)$ of semi-groups.

Employing a more general procedure of taking limits of sequences of operators (namely the limit inferior), we prove a generalization of the results of Miyadera [5] (Theorem 2 and Corollary 1 below). Therefore under rather mild conditions, convergence is established where it is possible that the limit does not exist in the sense of [5], [8]. We also note that convergence of $C_{0}$ semi-groups holds under weaker conditions than the one mentioned above (see Corollary 2 and the remarks below).

\section{$\S 1$. Preliminaries}

In what follows, $(X,\|\|$.$) is a Banach space, L(X)$ the space of bounded linear operators on $X$. For an arbitrary linear operator $A$ from $X$ to itself, $D(A), R(A)$ and $G(A)$ denote the domain, range, and the

*Research done at the MRC-University of Wisconsin under the sponsorship of the U.S. Army, Contract No. DAAG 29-80-C-0041 
graph of $A$, respectively. Occasionally we follow the practice of identifying an operator by its graph. We also denote the resolvent set of $A$ by $\rho(A)$ and the resolvent operator by $R(\lambda: A)=(\lambda I-A)^{-1}$.

Let $\left(X_{n},\|.\|_{n}\right), n=1,2, \ldots$ be a sequence of Banach spaces and let $P_{n}$ : $X \rightarrow X_{n}, n \in N$ be continuous linear maps such that for each $x$ in $X$

$$
\lim _{n \rightarrow \infty}\left\|P_{n} x\right\|_{n}=\|x\| .
$$

In this case the Banach-Steinhause theorem shows that there exists a constant $c>0$ such that

$$
\left\|P_{n} x\right\|_{n} \leq c\|x\|
$$

A sequence $\left(x_{n}\right), x_{n}$ in $X_{n}$ is said to converge to an element $x$ in $X$, written in

$$
\widetilde{\lim } x_{n}=x, \Longleftrightarrow \lim _{n \rightarrow \infty}\left\|P_{n} x-x_{n}\right\|_{n}=0
$$

We also consider sequences of operators $\left(A_{n}\right), A_{n}: X_{n} \rightarrow X_{n}$, and the limit of the sequence $\left(A_{n}\right)$, denoted by $\lim A_{n}$ (cf. [8] ), is an operator on $X$ whose domain consists of all $x$ in $X$ for which there exists a corresponding element $y$ such that $P_{n} x \in D\left(A_{n}\right)$ and $\widetilde{\lim } A_{n} P_{n} x=y$. A more general procedure of forming limits of sequences of operators is that of limit inferior denoted by $\lim \inf A_{n}$ or as we shall denote it by $\hat{A}$, it is defined (see also [4]) as follows :

$\lim \inf A_{n}=\left\{(x, y) \in X \times X\right.$ : there exists a sequence $\left(x_{n}\right), x_{n} \in D\left(A_{n}\right)$ such that, $\widetilde{\lim } x_{n}=x$ and $\left.\widetilde{\lim } A_{n} x_{n}=y\right\}$.

Considering $\lim \inf A_{n}$ as a function on $X$ it is, in general, multivalued and is an extension of the operator $\lim A_{n}$. The following set will also be useful in our investigation;

$D^{\circ}=\left\{x \in X\right.$ : there exists a sequence $\left(x_{n}\right), x_{n} \in D\left(A_{n}\right)$ such that $\widetilde{\lim } x_{n}=x$ and $\left.\sup _{n}\left\|A_{n} x_{n}\right\|<\infty\right\}$.

Next we review few facts about semi-groups of linear operators, for further details and information (refer to [2] and [9]). A semi-group of linear operators on $X$ is a mapping $T(t):(0, \infty) \rightarrow L(X)$ satisfying $T(t+$ $s)=T(t) T(s)$, for all $t, s>0$. It will be assumed here that $T(t)$ is strongly continuous on $(0, \infty)$. The infinitesimal operator of $T(t)$ is defined as usual by

$$
A_{0} x=\lim _{h \rightarrow 0+} h^{-1}(T(h)-I) x
$$

whenever the limit exists. In general, $A_{0}$ is an unbounded operator which need not even be closed. If the closure $\overline{A_{0}}$ exists, then it is called the infinitesimal generator (i.g.) of $T(t)$. The type of $T(t)$, denoted by $\omega$, 
is given by ;

$$
\omega=\liminf _{t>0} t^{-1} \log \|T(t)\| .
$$

If $T(t)$ is strongly continuous at $t=0$, then it is called a $C_{0}$ semigroup. In this case, there exists constants $M>0, \omega$ such that $\|T(t)\| \leq$ $M e^{\omega t}, t \geq 0$, and $A_{0}$ is closed (cf [2]). In the abscence of continuity at the origin we consider a more general class of semi-groups known as class $(1, A)$ (cf [2]), which we now define

DEFInITION. A strongly continuous semi-group $T(t), t>0$ is said to be of class $(1, A)$ iff the following conditions are satisfied:

(a) $\int_{0}^{1}\|T(t)\| d t<\infty$,
(b) $\lim _{\lambda \rightarrow \infty} \lambda \int_{0}^{\infty} e^{-\lambda t} T(t) x d t=x, \quad \Re e \lambda>\omega, x \in X$.

It follows from (a) that the integral in (b) exists and defines a bounded linear operator $R(\lambda)$ on $X$. Moreover the closure $\overline{A_{0}}$ exists and $R(\lambda$; $\left.\bar{A}_{0}\right)=R(\lambda)$, for $\Re e \lambda>\omega$. Finally we note that every $C_{0}$ semi-group is of class $(1, A)$ but that the converse is not true in general as indicated by the examples in [2], [7].

\section{$\S 2$. Convergence of Semi-groups.}

In what follows, the notation $\{T(t, A), t>0\}$ will be used to mean a semi-group $T(t)$ with i.g. $A$. For conditions $(*)$ and $(* *)$ below, we refer to [5].

THEOREM 1. For each $n$ in $N$, let $\left\{T\left(t ; A_{n}\right), t>0\right\}$ be a semi-group of linear operators on $X_{n}$ of class $(1, A)$. Let there exist constants $M$, $K>0$ and $\omega \geq 0$ such that

$$
\int_{0}^{\infty} e^{-\omega \xi}\left\|T\left(\xi ; A_{n}\right)\right\|_{n} d \xi \leq M, n \in N
$$

and

$$
\| \lambda R\left(\lambda ; A_{n} \|_{n} \leq K, \lambda \geq \omega, n \in N .\right.
$$

Further, assume that $D^{\circ}$ and $R\left(\lambda_{0} I-\hat{A}\right)$ are dense in $X$, for some $\lambda_{0}>\omega$. Then $\hat{A}$ is a densely defined single valued operator on $X$ with $\rho(\hat{A}) \supseteq$ $(\omega, \infty)$ and such that

$$
\lim _{\lambda \rightarrow \infty} \lambda R(\lambda ; \hat{A}) x=x, x \in X
$$

and moreover, for each $\lambda>\omega$ 


$$
\lim _{n \rightarrow \infty}\left\|R\left(\lambda ; A_{n}\right) P_{n} x-P_{n} R(\lambda ; \hat{A}) x\right\|_{n}=0, x \in X .
$$

ProOF. Let $\lambda_{0}>\omega$ be as in the hypothesis and let $x \in R\left(\lambda_{0} I-\hat{A}\right)$, then it is easy to verify that there exists a unique element $y \in X$ such that

$$
\lim _{n \rightarrow \infty}\left\|R\left(\lambda_{0} I ; A_{n}\right) P_{n} x-P_{n} y\right\|_{n}=0 .
$$

Hence we obtain a map $J\left(\lambda_{0}\right): R\left(\lambda_{0} I-\hat{A}\right) \rightarrow X$ defined by $J\left(\lambda_{0}\right) x=y$. Also (*) implies

$$
\| R\left(\lambda ; A_{n} \|_{n} \leq M, n \in N, \Re e \lambda>\omega .\right.
$$

Combining (4), (5) and the assumption that $R\left(\lambda_{0} I-\hat{A}\right)$ is dense, we conclude by continuity that $J\left(\lambda_{0}\right)$ may be extended to all of $X$ and furthermore

$$
\lim _{n \rightarrow \infty}\left\|R\left(\lambda_{0} ; A_{n}\right)^{\kappa} P_{n} x-P_{n} J\left(\lambda_{0}\right)^{\kappa} x\right\|_{n}=0, \kappa \in N, x \in X .
$$

where $J\left(\lambda_{0}\right)^{\kappa}$ is defined inductively on $\kappa$. Now each $\lambda>\omega$ for which (4) holds, yields an operator $J(\lambda)$ which satisfies (6). The following argument shows that $J(\lambda)$ exists for all $\lambda>\omega$. Let $\Lambda$ be the set of all numbers $\lambda>\omega$ for which $J(\lambda)$ is defined. We note in view of lemma 4.1 of [5] that the representation

$$
R\left(\lambda ; A_{n}\right)=\sum_{\kappa=0}^{\infty}\left(\lambda-\lambda_{0}\right)^{\kappa} R\left(\lambda_{0} ; A_{n}\right)^{\kappa+1}
$$

is valid for $\omega<\lambda<2 \lambda_{0}-\omega$. Using (6) and (7) one can verify that $\Lambda$ is both open and closed in $(\omega, \infty)$ and since $\lambda_{0} \in \Lambda$, we find that $\Lambda=(\omega, \infty)$.

Next we verify that $J(\lambda)$ is Abel summable, i.e.,

$$
\lim _{\lambda \rightarrow \infty} \lambda J(\lambda) x=x, x \in X \text {. }
$$

Let $x \in D^{\circ}$, and $\left(x_{n}\right)$ a sequence, $x_{n} \in D\left(A_{n}\right)$ such that $\widetilde{\lim } x_{n}=x$ and $\left\|A_{n} x_{n}\right\|_{n} \leq \mathrm{b}$. Then

$$
\begin{aligned}
\left\|P_{n}(\lambda J(\lambda) x-x)\right\|_{n} & \leq\left\|P_{n}(\lambda J(\lambda) x)-\lambda R\left(\lambda ; A_{n}\right) P_{n} x\right\|_{n} \\
& +\left\|\lambda R\left(\lambda ; A_{n}\right) P_{n} x-\lambda R\left(\lambda ; A_{n}\right) x_{n}\right\|_{n} \\
& +\left\|\lambda R\left(\lambda ; A_{n}\right) x_{n}-x_{n}\right\|_{n}+\left\|x_{n}-P_{n} x\right\|_{n}
\end{aligned}
$$

and the 3rd term on the right hand side is dominated by

$$
\begin{aligned}
\left\|R\left(\lambda ; A_{n}\right) A_{n} x_{n}\right\|_{n} & =\frac{1}{\lambda}\left\|A_{n} x_{n}+A_{n} R\left(\lambda ; A_{n}\right) A_{n} x_{n}\right\|_{n} \\
& \leq \frac{1}{\lambda}\left\|A_{n} x_{n}\right\|_{n}\left(1+\left\|A_{n} R\left(\lambda ; A_{n}\right)\right\|_{n}\right)
\end{aligned}
$$




$$
\leq \frac{b}{\lambda}(2+K)
$$

Passing to the limit as $n \rightarrow \infty$ and then as $\lambda \rightarrow \infty$, we obtain $\lim _{\lambda \rightarrow \infty} \lambda J(\lambda) x=$ $x$. Since $D^{\circ}$ is dense in $X$, the conclusion follows by the BanachSteinhaus argument.

To show that $\hat{A}$ is single valued, assume that $(0, y) \in \hat{A}$ and let $x_{n} \in$ $D\left(A_{n}\right), n \in N$ be such that $\widetilde{\lim } x_{n}=0$ and $\widetilde{\lim } A_{n} x_{n}=y$. Put $y_{n}=$ $\left(\lambda I-A_{n}\right) x_{n}(\lambda>\omega)$. Then $\lim _{n \rightarrow \infty}\left\|y_{n}+P_{n} y\right\|_{n}=0$, hence

$$
\begin{aligned}
\lim _{n \rightarrow \infty}\left\|R\left(\lambda ; A_{n}\right) P_{n} y\right\|_{n} & \leq \lim _{n \rightarrow \infty}\left\|R\left(\lambda ; A_{n}\right) P_{n} y+R\left(\lambda ; A_{n}\right) y_{n}\right\|_{n} \\
& +\lim _{n \rightarrow \infty}\left\|R\left(\lambda ; A_{n}\right) y_{n}\right\|_{n}=0 .
\end{aligned}
$$

From this and (6) we find that $J(\lambda) y=0$ for all $\lambda>\omega$, which implies $y=0$.

It remains to show that $J(\lambda)=R(\lambda ; \hat{A})$, for $\lambda>\omega$. Let $x \in X$ and $y=$ $J(\lambda) x$. Then, as before, $\lim _{n \rightarrow \infty}\left\|R\left(\lambda ; A_{n}\right) P_{n} x-P_{n} y\right\|_{n}=0$ and with $y_{n}=R(\lambda$; $\left.A_{n}\right) \mathrm{P}_{n} \mathrm{x}$ we find that $\widetilde{\lim } y_{n}=y$ and $\widetilde{\lim } A_{n} y_{n}=\lambda y-x$. Therefore $y \in D(\hat{A})$ and $\hat{A} y=\lambda y-x$, which yields $x=(\lambda I-\hat{A}) J(\lambda) x$. Similarly, one verifies that $J(\lambda)(\lambda I-\hat{A}) x=x$, for $x \in D(\hat{A})$

We shall use the following notation for convenience;

$$
\begin{aligned}
& H(\omega)=\{\lambda: \Re e \lambda>\omega\}, \\
& S(\omega)=\rho(\hat{A}) \cap H(\omega) .
\end{aligned}
$$

Proposition 1. Suppose that $\left\{T\left(t ; A_{n}\right) ; t>0\right\}$ satisfies (*) and (**) such that $D^{\circ}$ and $R\left(\lambda_{0} I-\hat{A}\right)$ are dense in $X$ for some $\lambda_{0}>\omega$, then the following assertions hold:

(a) For each $\lambda \in S(\omega)$

$$
R(\lambda ; \hat{A})=\lim \inf R\left(\lambda ; A_{n}\right)
$$

and

$$
\| R(\lambda ; \hat{A} \| \leq M
$$

(b) $H(\omega) \subseteq \rho(\hat{A})$,

(c) $R(\lambda ; \hat{A})^{\kappa}=\liminf R\left(\lambda ; A_{n}\right)^{\kappa},(\kappa \geq 1), \lambda \in H(\omega)$,

(d) For each $z \in D\left(\hat{A}^{2}\right)$ there exists a sequence $\left(z_{n}\right), z_{n} \in D\left(A_{n}^{2}\right)$ such that

$\widetilde{\lim } z_{n}=z, \widetilde{\lim } A_{n} z_{n}=\hat{A} z$ and $\widetilde{\lim } A_{n}^{2} z_{n}=\hat{A}^{2} z$, in particular $\lim \inf A_{n}^{2} \supset \hat{A}^{2}$.

Proof. (a) Let $\lambda \in S(\omega)$ and let $x \in X, y \in D(\hat{A})$ be such that $x=$ $\lambda y-\hat{A} y$. Then, by definition, there exists $y_{n} \in D\left(A_{n}\right), n \in N$ such that $\widetilde{\lim } y_{n}=\mathrm{y}$ and $\widetilde{\lim } A_{n} y_{n}=\hat{A} y$. Put $x_{n}=\lambda y_{n}-A_{n} y_{n}$. Then, clearly $\widetilde{\lim } x_{n}=x$ 
and $\widetilde{\lim } R\left(\lambda ; A_{n}\right) x_{n}=\mathrm{R}(\lambda ; \hat{A}) x$, while (9) follows from (8) and (5).

(b) Let $x \in D(\hat{A}), y=\hat{A} x$ and let $x_{n} \in D\left(A_{n}\right), n \in N$ be such that $\widetilde{\lim } x_{n}=\mathrm{x}$ and $\widetilde{\lim } A_{n} x_{n}=\hat{A} x$. Then, by (5)

$$
\left\|x_{n}\right\|_{n} \leq K\left\|\lambda_{0} x_{n}-A_{n} x_{n}\right\|_{n} .
$$

Passing to the limit, we get

$$
\|x\| \leq M\left\|\lambda_{0} x-\hat{A} x\right\|
$$

and since by assumption $R\left(\lambda_{0} I-\hat{A}\right)$ is dense, we find that $\lambda_{0} \in S(\omega)$. Now, let $\lambda_{n} \rightarrow \lambda$, where $\lambda_{n} \in S(\omega)$ and $\lambda \in H(\omega)$. Using (9), we find that for each $x \in X$,

$$
\| \lambda-\hat{A}) R\left(\lambda_{n} ; \hat{A}\right) x-x\|=\|\left(\lambda-\lambda_{n}\right) R\left(\lambda_{n} ; \hat{A}\right) x\left\|\leq M \mid \lambda-\lambda_{n}\right\| x \|,
$$

showing that $R(\lambda I-\hat{A})$ is dense in $X$. Replacing $\lambda_{0}$ in the preceding argument by $\lambda$, we have that $\lambda \in S(\omega)$. Thus $S(\omega)$ is a nonvoid closed and open subset of $H(\omega)$, hence is equal to it.

(c) follows from (a) and (b) by induction

(d) Let $z \in D\left(\hat{A}^{2}\right)$ and let $x \in X$ be such that $z=R\left(\lambda_{0} ; \hat{A}\right)^{2} x$. Put $y=$ $R\left(\lambda_{0} ; \hat{A}\right) x, x_{n}=P_{n} x, y_{n}=R\left(\lambda_{0} ; A_{n}\right) x_{n}$ and $z_{n}=R\left(\lambda_{0} ; A_{n}\right) y_{n}$. Using (a) we find that

$$
\begin{aligned}
& \widetilde{\lim } y_{n}=R\left(\lambda_{0} ; \hat{A}\right) x=y \text { and } \widetilde{\lim } z_{n}=R\left(\lambda_{0} ; \hat{A}\right) y=z \text {. Thus } \\
& \widetilde{\lim }\left(\lambda_{0} z_{n}-A_{n} z_{n}\right)=\lim y_{n}=y=\lambda_{0} z-\hat{A} z \text {, which implies } \\
& \widetilde{\lim } A_{n} z_{n}=\hat{A} z \text {. Similarly } \\
& \widetilde{\lim }\left(\lambda_{0} y_{n}-A_{n} y_{n}\right)=\widetilde{\lim } x_{n}=x=\lambda_{0} y-\hat{A} y \text { implies } \\
& \lim A_{n} y_{n}=\hat{A} y \text {. Hence } \\
& \widetilde{\lim } A_{n}\left(\lambda_{0} z_{n}-A_{n} z_{n}\right)=\hat{A}\left(\lambda_{0} z-\hat{A} z\right) \text { and } \widetilde{\lim } A_{n}^{2} z_{n}=\hat{A}^{2} z .
\end{aligned}
$$

We now state the main result

THEOREM 2. For each $n$ in $N$ let $\left\{T\left(t ; A_{n}\right), t>0\right\}$ be a semi-group of class $(1, A)$ on $X_{n}$ such that conditions (*) and (**) are satisfied. Then the following assertions are equivalent:

(i) There exists a semi-group $T(t)$ of class $(1, A)$ defined on $X$ such that for each $x$ in $X$ and $x_{n}$ in $X_{n}, n=1,2,3, \ldots$

$$
\widetilde{\lim } x_{n}=x \Rightarrow \widetilde{\lim } T\left(t ; A_{n}\right) x_{n}=T(t) x, t>0
$$

uniformly on compact subsets of $(0, \infty)$,

(ii) $D^{\circ}$ and $R\left(\lambda_{0} I-\hat{A}\right)$, for some $\lambda_{0}>\omega$ are dense in $X$.

In either case $\hat{A}$ is the i.g. of $T(t)$.

PROOF. (i $\Longrightarrow$ (ii) 
Assume that there exists a semi-group $T(t)$ of class $(1, A)$ satisfying (10) with i.g. $A$ and let $x \in X, x_{n} \in X_{n},(n \geq 1)$ be such that $\widetilde{\lim } x_{n}=x$. Then for sufficiently large $\lambda$

$$
\begin{aligned}
& \lim _{n \rightarrow \infty}\left\|R\left(\lambda ; A_{n}\right) x_{n}-P_{n} R(\lambda ; A) x\right\|_{n} \\
& \leq \lim _{n \rightarrow \infty} \int_{0}^{\infty} e^{-\lambda t}\left\|T\left(t ; A_{n}\right) x_{n}-P_{n} T(t) x\right\|_{n} d t=0 .
\end{aligned}
$$

Fix a large $\lambda$ and let $x \in D(A)$. Then there exists $y \in X$ such that $\lambda x-$ $A x=y$. Letting $y_{n}=P_{n} y$ and $x_{n}=R\left(\lambda ; A_{n}\right) y_{n}$, we have that $\widetilde{\lim } x_{n}=x$, $\widetilde{\lim } A_{n} x_{n}=\widetilde{\lim }\left(\lambda x_{n}-y_{n}\right)=\lambda x-y=A x$ and so $A \subset \hat{A}$. Since $T(t)$ is of class $(1, A), R(\lambda I-A)$ and $D(A)$ are both dense in $X$, hence the same is true for $R(\lambda I-\hat{A})$ and $D^{\circ}$. Now it follows from this and (3) that $A=\hat{A}$.

(ii) $\Longrightarrow($ i )

From Theorem 1 and Proposition $1 \hat{A}$ is a closed operator whose resolvent $R(\lambda ; \hat{A})$ is bounded in the half plane $H(\omega)$. Therefore, in view of a lemma of Hille and Phillips (cf. [2]) there exists $\gamma>\omega$ such that

$$
Y(t ; z)=z+t \hat{A} z+\frac{1}{2 \pi i} \int_{\gamma-i \infty}^{\gamma+i \infty} e^{\lambda t} R(\lambda ; \hat{A}) \hat{A}^{2} z \frac{d \lambda}{\lambda^{2}}
$$

defines for each $z \in D\left(\hat{A}^{2}\right)$ a continuous function on $t \geq 0$ with $Y(0 ; z)=z$. Similarly, since each $T\left(t ; A_{n}\right)$ is of class $(1, A)$ with $H(\omega) \subseteq \rho\left(A_{n}\right)$,

$\left[A_{n}^{2}\right]$.

$$
T\left(t ; A_{n}\right) w=w+t A_{n} w+\frac{1}{2 \pi i} \int_{\gamma-i \infty}^{\gamma+i \infty} e^{\lambda t} R\left(\lambda ; A_{n}\right) A_{n}^{2} w \frac{d \lambda}{\lambda^{2}}, w \in D
$$

Now let $z \in D\left(\hat{A}^{2}\right)$ and let $\left(z_{n}\right)$ be a sequence as given by Proposition 1 -(d), replacing $w$ by $z_{n}$ in (12) and passing to the limit as $n \rightarrow \infty$, we find that the lst and 2nd terms in the right hand side of (12) tend to those in the right hand side of (11). To see that the integral in (12) also converges to the integral in (11), we note firstly by Proposition 1 that

$$
\widetilde{\lim } R\left(\lambda ; A_{n}\right) A_{n}^{2} z_{n}=R(\lambda ; \hat{A}) \hat{A}^{2} z
$$

and secondly that there exist positive constants $M, \alpha$ independent of $n$ such that the integrand in (12) is dominated in norm by $\alpha M e^{\gamma t} /|\lambda|^{2}$, and this is integrable over the line $\gamma$. In conclusion we find that

$$
\widetilde{\lim } T\left(t ; A_{n}\right) z_{n}=Y(t ; z), t \geq 0, z \in D\left(\hat{A}^{2}\right) .
$$

Furthermore, using Theorem 7.7.4 of [2] and (13) we have

$$
\|Y(t ; z)\| \leq\left(C_{1} M^{2} e^{\gamma t} / t^{2}\right)\|z\|, t>0, z \in D\left(\hat{A}^{2}\right),
$$


where $C_{1}$ is a constant. Therefore since $D\left(\hat{A}^{2}\right)$ is dense in $X, Y(t ; z)$ has a unique bounded extension $T(t)$ on $X$ which satisfies (14).

It is readily verified that

$$
\widetilde{\lim } T\left(t ; A_{n}\right) P_{n} z=T(t) z, z \in X,
$$

uniformly on compact subsets of $(0, \infty)$ and that $T(t)$ has the semi-group property. To verify that it is of class $(1, A)$, we note from

$$
\begin{aligned}
& \|T(t)\| \leq \liminf _{n} \| T\left(t ; A_{n} \|_{n}\right. \text { that } \\
& \int_{0}^{\infty} e^{-\omega t}\|T(t)\| d t \leq \liminf _{n} \int_{0}^{\infty} e^{-\omega t} \| T\left(t ; A_{n} \| d t \leq M .\right.
\end{aligned}
$$

Thus the integral

$$
R(\lambda) x=\int_{0}^{\infty} e^{-\lambda t} T(t) x d t
$$

exists for all $x \in X, \Re e \lambda>\omega$, and as before

$$
\lim _{n \rightarrow \infty}\left\|R\left(\lambda ; A_{n}\right) P_{n} x-P_{n} R(\lambda) x\right\|_{n}=0 .
$$

Hence $R(\lambda)=R(\lambda ; \hat{A})$, in particular recalling (2), we see that $T(t)$ is of the class $(1, A)$ and $\hat{A}$ is the i.g. of $T(t)$.

COROLlary 1. Suppose that the hypotheses of Theorem 2 are fullfilled. Then the following are equivalent:

(i) There exists a semi-group $T(t)$ of class $(1, A)$ defined on $X$ such that for each $x$ in $X$ and $x_{n}$ in $X_{n}, n=1,2,3, \ldots$,

$$
\widetilde{\lim } x_{n}=x \Rightarrow \widetilde{\lim } T\left(t ; A_{n}\right) x_{n}=T(t) x, t>0,
$$

uniformly on compact subsets of $(0, \infty)$,

(ii) There exists a densely defined operator $A$ such that $A \subset \liminf A_{n}$ and such that $R(\lambda I-A)$ is dense for some $\lambda>\omega$.

In either case the closure $\bar{A}$ is the i.g. of $T(t)$.

COROLlary 2. If for each $n$ in $N, T\left(t ; A_{n}\right)$ is a $C_{0}$ semi-group satisfying conditions (*) and (**), then the conclusion of Theorem 2 and or Cor 1 holds.

\section{REMARKS}

1. Replacing $A$ by $\lim A_{n}$ in Corollary 1, one obtains the results of [5] which also extends the results of [8] on this point.

2. We note, in Corollary 2 that the limit semi-group need not be of class

$C_{0}$. In fact if it is a $C_{0}$ semi-group, then 


$$
\left\|T\left(t ; A_{n}\right)\right\|_{n} \leq M e^{\omega t}, \quad t \geq 0,
$$

where $M$ and $\omega$ are independent of $n$.

\section{References}

[1] M. HASEgawa, A note on the convergence of semi-groups of operators. Proc. Japan Acad. 40 (1964), 262-266.

[2] E. Hille and R. S. PHILliPS, Functional analysis and semi-groups, rev. ed., Amer. Math. Soc. Colloq. Publ., Vol. 31, Providence, R. I., 1957.

[ 3 ] T. KATO, "Purturbation Theory for Linear Operators" Berlin-Heidelberg-New YorkTokyo, Springer 1984.

[4] T. G. KURTZ, Extensions of Trotter's operator semi-group approximation theorems, J. Func. Anal. 3 (1969), 354-375.

[ 5 ] I. MIYADERA, Perturbation theory for semi-groups of operators, Sagaku., 20 (1968), 14 -25 .

[6] S ÔHARU, On the convergence of semi-groups of operators, Proc. Japan Acad., 42 (1966), 880-884.

[ 7 ] R. S. PHILLIPS, An inversion formula for the Laplace transforms and semi-groups of linear operators, Annals of Math. Vol. 59, No. 2 (1954), 325-356.

[8] H. F. TROTTER, Approximation of semi-groups of operators, Pac. Jour. Math., 8 (1958), 887-919.

[ 9 ] K. YOSIDA, "Functional Analysis" (6th edition) Springer Verlag (1980).

Mathematics Department

Kuwait University 